\title{
El climaterio. ¿Cambio fisiológico o enfermedad?
}

\author{
Dr. Alfredo Jácome-Roca, F.A.C.P.*
}

RESUMEN. El climaterio es un fenómeno fisiológico que tiene connotaciones francamente patológicas debido a la estrógenodeficiencia asociada. La menopausia o menstruación final separa un período previo relativamente corto, la premenopausia, de otro más prolongado, la postmenopausia, con alteraciones hormonales y metabólicas.

Desde el punto de vista endocrino se observa una caída en el estradiol asociada a elevación en las gonadotropinas, particularmente de la FSH. El principal estrógeno circulante será entonces el sulfato de estrona, resultado de la aromatización periférica de androstenediona. Su concentración es mayor en las obesas pero es variable, y puede determinarse indirectamente a través de la prueba de progesterona.

El climaterio tiene manifestaciones a corto y a largo plazo. Los síntomas vasomotores son comunes y muy molestos, al igual que las manifestaciones urogenitales (dispareunia, incontinencia urinaria, "síndrome uretral"), las psicológicas, sexuales y los cambios de la piel; la estrógenoterapia es frecuentemente prescrita con buenos resultados.

Las alteraciones metabólicas que se observan en el transcurso de los años tienen mayor importancia desde el punto de vista de la morbi-mortalidad. La disminución de la masa ósea observada en la osteoporosis postmenopáusica lleva a fracturas, cuadros dolorosos, deformidades y eventual invalidez y/o muerte; la aterosclerosis es sin embargo el aspecto más importante (aumento en la incidencia de enfermedad coronaria y accidentes cerebro-vasculares). El efecto benéfico es probablemente de origen multifactorial pero se debe destacar la reversión del patrón lipídico aterogénico que se ve en la postmenopausia.

Aunque hay distintas formas de tratar cada una de las alteraciones observadas en el climaterio, la estrógenoterapia es la única que previene y/o corrige todas ellas. Existen contraindicaciones clásicas para el uso de los estrógenos, pero el principal debate está relacionado con los cánceres femeninos. El del endometrio se combate de manera efectiva con la adición de un progestágeno, el cual no es necesario usarlo en mujeres histerectomizadas. En cuanto al cáncer de seno, tumor de mayor incidencia en esas etapas postreras de la vida, su incidencia no parece afectarse con el uso de estrógenos a corto plazo (3, 5 y hasta 10 años) y sólo parece tener un impacto menor, un ligero incremento en la incidencia, cuando se usa por más de 10 años. Sin embargo si se compara la mortalidad global entre usuarias y no usuarias de estrógenos, ésta es significativamente menor en las primeras, particularmente con el uso actual y a mayor plazo. Esto probablemente se debe a que la principal causa de muerte en la climatérica es la enfermedad coronaria, que se reduce por la cardioprotección ofrecida por los estrógenos. (Rev Col Obstet Ginecol 1991; 42(3): 187-196).

PALABRAS CLAVES: Climaterio, estrógenos, osteoporosis, aterosclerosis, carcinoma endometrial, cáncer de seno, cardioprotección.

SUMMARY. The climacteric is a physiologic phenomenon with pathologic implications, due to the associated estrogen defficiency. Menopause or final menstrual period separates a rather short stage, premenopause, from a longer one, the postmenopause, characterized by hormonal and metabolic derangements.

From the endocrine point of view, a fall in estradiol levels is observed; concomitantly, there is a rise in serum and urinary gonadotrophins, particularly in FSH. Estrone sulphate will become the main circulating estrogen, obtained from peripheral conversion of androstenedione. Its concentration is greater in obese women but is variable, and can indirectly be measured through the progesterone test.

There are short-term and long-term manifestations of the climacteric. Vasomotor symptoms are common and bothersome, as well as urogenital complaints (dyspareunia, urinary incontinence, "urethral syndrome"), psychological and sexual problems, skin changes; estrogen replacement therapy (ERT) is frequently prescribed with good results.

Metabolic derangements observed through the years are more important from the morbidity and mortality point of view. A decrease in bone mass observed in postmenopausal osteoporosis leads to bone fractures, painful syndromes, deformities and eventual disability and/or death; atherosclerosis is however the most important trouble (increase in the incidense of coronary heart disease and cerebro-vascular accidents).

Beneficial effects are probably multifactorial in origin but the reversal in the postmenopausal atherogenic lipid profile should be emphasized.

Though there are different ways to treat each one of the pathological manifestations of the climacteric, ERT is the only one that prevents and/or.corrects them all. There are classic contraindications for the use of estrogens, but main debàte is centered on female cancer. Endometrial cancer is effectively prevented with the concomitant use of a progestogen, which needs not to be used in histerectomized women. In regard to breast cancer, with a greater incidence in last decades of life, short-term ERT $(3,5$, or up to 10 years) does not seem to increase the incidence, long-term use (10 or more years) may increase the incidence somewhat, an impact considered by some to be minimal. If global mortality between users and non-users is compared, this is significantly lower in the former, particularly with current or longer-term use. This probably reflects a cardioprotective effect of estrogens, having in mind that the main cause of death in the climacteric is coronary heart disease.

KEY WORDS: Climacteric, estrogen, osteoporosis, atherosclerosis, endometrial carcinoma, breast cancer, cardioprotection.

\footnotetext{
* Miembro de Número, Academia Nacional de Medicina, expresidente Socie-

dad Colombiana de Endocrinología, Santafé de Bogotá, D.C. A.A. 8620.
} 


\section{Introducción}

Tres etapas tiene la vida de la mujer desde el punto de vista endocrinológico, que además coinciden muy bien con diferentes aspectos psicológicos, físicos y socio-económicos, propios de cada período.

La infancia o etapa prepuberal cursa con los niveles hormonales muy bajos; el eje hipotálamo hipófisis-ovarios no se ha desarrollado y el útero se mantiene infantil, al igual que el aspecto somático y psicológico de la niña. La menarquia marca el inicio de la etapa reproductiva: la aparición de las características sexuales secundarias y de los ciclos menstruales con la secreción de gonadotropinas, estrógenos y progesterona, la ovulación, la vida de pareja y los embarazos.

El climaterio, un término comúnmente usado para englobar la premenopausia, la menopausia y la postmenopausia, fase de envejecimiento de la mujer que marca la transición de la vida reproductiva a la infértil, es a la vez temido $\mathrm{y}$ ansiado (1).

Así como la menarquia indica la primera regla, la menstruación final señala la menopausia. La edad promedio de aparición de la menopausia en los Estados Unidos es de aproximadamente 50 años; como en ese país la esperanza femenina de vida es de 80 , se puede afirmar que la mujer vivirá un $30 \%$ de su experiencia vital en el período postmenopáusico (2).

En el pasado se percibía la época postmenopáusica como una fatalidad que tanto la paciente como sus familiares debían soportar con resignación; era una etapa fisiológica, la final, que marcaba la declinación de la mujer. Pasaba ella a ser la "abuela", la "jubilada", algo así como la que tenía su sitio en el desván de los trastos viejos. En muchas sociedades se la veía como una criatura sin actividad sexual, inestable, canosa y con arrugas, que se encontraba en espera del final de sus días.

Hoy muchos médicos están de acuerdo en que la menopausia es un estado de hormono-deficiencia; algunos, sin embargo, la siguen considerando un evento fisiológico y así pues sería una intrusión alterar el proceso natural de envejecimiento. El "quid" del manejo terapéutico dependerá del concepto "etapa fisiológica vs. deficiencia hormonal". El médico decidirá entonces si quiere o no tratar los síntomas vasomotores, la atrofia urogenital y las manifestaciones psicogénicas, si prefiere no involucrarse por el "temor al cáncer", si considera importante prevenir la osteoporosis o disminuir la mortalidad por enfermedad coronaria y por otras alteraciones vasculares arterioescleróticas.

La decisión no es fácil, pues la suplencia con estroprogestágenos tiene algunas contraindicaciones y precauciones, pero tiene también muchas ventajas. Es cuestion de poner en la balanza riesgo vs. beneficio de la "Terapia de Suplencia Estrogénica", tema sobre el cual se escriben muchos artículos en las revistas médicas de hoy.

Con la asociación de un progestágeno al régimen de estrógenos, el riesgo de cáncer endometrial desapareció. La controversia sobre la terapia de suplencia estrogénica tiende en los años 90 a ser favorable, cuando se está recobrando la popularidad vista en la década de los $60 \mathrm{y}$ se ha aprendido a evitar las complicaciones observadas en los 70 .

Gambrell (1), considerado por muchos como el padre de la terapia de suplencia hormonal, afirma que "hay un reco- nocimiento creciente de que la deficiencia estrogénica debe ser tratada tan vigorosamente como cualquier otra endocrinopatía y sin limitaciones de tiempo". Se habla ya de terapias continuas en vez de cíclicas, administradas durante años en vez de meses. El diagnóstico del climaterio se basa en las características clínicas de oleadas de calor con enrojecimiento, sudoración nocturna, irritación y sequedad vaginales y manifestaciones psicogénicas que con el tiempo se asociarán a problemas de osteoporosis y enfermedad coronaria y/o arterioesclerosis. Desde el punto de vista de laboratorio, se puede observar una citología vaginal característica de deprivación estrogénica, compuesta principalmente por células basales, aunque en muchos casos esta no es una prueba muy confiable; habrá una elevación marcada en las gonadotropinas, hasta de 13 veces en la FSH y de tres veces en la $\mathrm{LH}$, con un rango de 75 a $200 \mathrm{mU} / \mathrm{ml}$ para la primera y de 60 a $90 \mathrm{mU} / \mathrm{ml}$ para la segunda. El estradiol (E2) disminuirá de $120 \mathrm{pg} / \mathrm{ml}$ a sólo $13 \mathrm{pg} / \mathrm{ml}$. El principal estrógeno circulante será entonces el sulfato de estrona, un estrógeno conjugado formado por la conversión periférica de androstenediona por acción de una aromatasa.y que alcanzará un nivel promedio de $178 \mathrm{pg} / \mathrm{ml}$. Los andrógenos suprarrenales dehidroepiandrosterona (DHEA) y su sulfato (DHEA-S) disminuyen entre un 20 y $40 \%$. Una prueba de progesterona, parenteral $\mathrm{u}$ oral, que no conlleve posteriormente a una hemorragia de supresión, indicará la necesidad de estrógenoterapia, pues hay ausencia de una fase proliferativa adecuada $(3,4)$.

Pasemos ahora a revisar tanto las manifestaciones como las complicaciones asociadas al síndrome climatérico.

\section{Síntomas vasomotores}

Las oleadas de calor con enrojecimiento (bochornos) y la sudoración nocturna son frecuentes en la menopausia; tienen un comienzo lento y aumentan a medida que declinan los estrógenos séricos (5). Un $80 \%$ experimentan síntomas por más de un año y $50 \%$ por más de cinco años; al menos la mitad requiere tratamiento (2). Su severidad y frecuencia son muy variables. El bochorno menopáusico es una respuesta circulatoria evanescente: al comenzar los síntomas, hay un aumento rápido y marcado en el flujo sanguíneo de la mano por algunos minutos, luego un aumento menor y más corto pero sostenido tanto en el flujo sanguíneo del antebrazo como en la frecuencia del pulso sin que haya cambios en las cifras de tensión arterial. Esta vasodilatación periférica está confinada a los vasos sanguíneos de la piel y contrasta con los cambios cardiovasculares observados durante una reacción emocional. En los hombres con hipogonadismo pueden ocurrir bochornos virtualmente idénticos (6). La respuesta vascular se parece a la observada en el calentamiento indirecto y sugiere que haya una activación inapropiada en los mecanismos para disipar el calor, es decir en la función nerviosa termorreguladora, tal vez dependiente de alguna sustancia producida tanto en ovario como en testículo y que tenga efecto sobre el hipotálamo. Se ha mostrado una asociación entre la liberación pulsátil de LH y la presencia de los bochornos pero estos también pueden ocurrir en hipofisectomizados. Las pacientes con disgenesia gonadal y LH elevada sufren de calores sólo cuando reciben terapia de suplencia estrogénica y ésta es luego suspendida. Los enrojecimientos pueden empezar a desaparecer a medida 
que el hipotálamo y el Sistema Nervioso Autónomo se van ajustando a los niveles bajos de estrógenos (1).

Los bochornos pueden aparecer espontáneamente o ser provocados por el calor o las bebidas calientes, el alcohol o el estrés mental. Estas oleadas de calor tienen un impacto deletéreo sobre el sueño; el insomnio resultante y la fatiga crónica pueden entonces contribuir a la inestabilidad emocional. La administración de estrógenos disminuye significativamente la frecuencia de los bochornos (7). En un estudio realizado en la Argentina por Figueroa-Casas (8), 74 mujeres menopáusicas con bochornos y al menos algún otro síntoma como sudoración, palpitaciones, nerviosismo leve y vaginitis atrófica, fueron tratadas con $0.625 \mathrm{mg}$ de estrógenos equinos conjugados durante tres semanas ( $y$ una semana libre) asociados con acetato de medroxiprogesterona (10 mg diarios durante diez días) por un período mayor de tres años. La respuesta en cuanto a estos síntomas fue buena en el $86.4 \%$ de los casos y parcial en un $9.5 \%$ adicional. Siendo esta una terapia cíclica, la gran mayoría de las pacientes presentaron hemorragia por supresión $(82.5 \%)$ o en otras, de desprendimiento. Seis pacientes que presentaban hiperplasia endometrial antes del tratamiento, revirtieron durante esta administración secuencial de estroprogestágenos.

Hay pues poca duda de que los síntomas causados por inestabilidad vasomotora tales como bochornos y sudoración nocturna pueden aliviarse con el tratamiento estrógenico (9); se han usado también otros agentes: progestágenos, clonidina y tranquilizantes, ninguno según Hammond, tan efectivos como los estrógenos (7). Recientemente se han usado también antagonistas de la dopamina del tipo ortopramidas. En general, se ensayan drogas que actúan sobre los sistemas adrenérgico o dopaminérgico o los sedantes, y estos medicamentos se dispersan si hay objeciones a la terapia hormonal (10).

\section{Manifestaciones urogenitales, psicológicas, sexuales y en la piel, observadas en el climaterio}

La deprivación estrogénica lleva a una atrofia del epitelio genital, con cambios en la cantidad de líquido vaginal, niveles de $\mathrm{pH}$ y flujo sanguíneo vaginal (1). Estos cambios también se observan en estructuras contiguas ricas en receptores estrogénicos y que tienen un origen embriológico común como el útero, uretra y trígono vesical, que responden a dosis menores de estrógenos de las que se requieren para el control de los síntomas vasomotores, usando bien la vía oral o la tópica (crema vaginal) (11). La vulva puede también atrofiarse pero responde pobremente a la terapia estrogénica. A medida que avanza la edad, la deprivación estrogénica es más notoria, pero si tenemos en cuenta que el estrógeno endógeno predominante en la menopausia es la estrona, pueden haber marcadas diferencias individuales debido a la variación en la producción del precursor androgénico androstenediona (proveniente más que todo de las suprarrenales) y también en el porcentaje de conversión a estrona (12). Las manifestaciones clínicas de estos cambios pueden ser la dispareunia, incontinencia urinaria, vaginitis infecciosa o el "síndrome uretral" (uretritis abacteriana recurrente), con disuria, poliaquiuria y tenesmo vesical (3). La vagina se distensiona con un poco de dificultad, en particular si los coitos son infrecuentes y los tejidos de sostén de la pelvis se relajan aunque este tipo de problemas que conduce a prolapsos, tienen más que ver con la edad, raza, paridad, predisposición genética y aumento de la presión abdominal.

Los efectos psicológicos y sexuales de la menopausia no se pueden enfocar de una manera simplista. No está claro que haya una mayor incidencia de la depresión; aunque los estrógenos no tienen efecto antidepresivo, sí pueden mejorar el bienestar general de la paciente. Los estrógenos podrían jugar un papel importante en la modificación del metabolismo de la dopamina y de la serotomina en el cerebro con una mejoría del estado de ánimo (que algunos han llamado efecto "mental tónico"). Por otro lado, el insomnio $\mathrm{y}$ el cansancio son frecuentemente informados en la menopausia. Es llamativa la asociación de oleadas de calor y el despertar nocturno; se ha visto que los estrógenos mejoran los patrones de sueño y aumentan la duración del sueño REM, lo que teóricamente podría disminuir algunos de los trastornos psicológicos vistos en el climaterio (3).

En cuanto a la esfera sexual, además de los fenómenos antes descritos y de la menor distensibilidad y lubricación vaginal, se ha visto que también hay modificaciones en la estimulación sensorial y en el flujo sanguíneo como consecuencia de la disminución de los niveles de estrógenos; dentro del contexto de todos estos cambios podrían englobarse los informes de mayor incidencia de dispareunia, vaginismo, disminución de la sensibilidad del clítoris, disminución en la frecuencia e intensidad del orgasmo, disminución de la libido y de la frecuencia del coito. Muchos de estos fenómenos revierten con el uso de estrógenos aún sin usar andrógenos, hormonas que pueden aumentar la libido en ambos sexos (13). Durante el proceso de envejecimiento se empieza a observar una atrofia cutánea, pues el número de células de la piel disminuye progresivamente durante el envejecimiento hasta la muerte. Se han determinado receptores estrógénicos en dermis y epidermis y la terapia estrogénica local produce una mejor elasticidad de la piel, mejoría del tejido colágeno y del cabello. Una formulación deliberada de estrógenos para estos problemas podría considerarse, mas no su uso cosmetológico indiscriminado en cremas de libre venta (14).

\section{Osteoporosis post-menopáusica}

La osteoporosis, definida como una disminución absoluta de la cantidad de hueso y que lleva fracturas después de mínimos traumas, es la enfermedad metabólica ósea más común, particularmente en la mujer. Aunque el volumen de los huesos osteoporóticos es esencialmente normal, hay una pérdida de masa ósea por unidad de volumen, también con pérdida en matriz ósea y calcio.

La porosidad ósea está aumentada y la masa ósea se encuentra por debajo del umbral donde comienzan a verse fracturas, cuya incidencia es alta en mujeres de más de 65 años. La osteoporosis es responsable de 1.200.000 fracturas al año en Norteamérica, la mitad de ellas localizadas en las vértebras y las restantes en caderas, muñecas (fractura de Colles) y en otros huesos de las extremidades. En las ancianas es común la fractura de cabeza de fémur, que puede ser fatal en un porcentaje de estas pacientes (15).

La remodelación ósea es una secuencia coordinada de eventos celulares que son estrechamente regulados por agentes elaborados tanto sistémica como localmente. Entre las células participantes están los osteoclastos, osteoblastos y 
sus precursores, osteocitos, las células de lineamiento óseo (osteoblastos en descanso) y otras células. Los agentes de acción sistémica tipo paratohormona y vitamina D3, pueden activar el ciclo de remodelación, pero debe haber un mecanismo para dirigir esos mensajes sistémicos a sitios específicos, un estado local de alistamiento o receptividad. Las fuerzas mecánicas y eléctricas pueden aumentar la posibilidad estadística de que la resistencia a la activación pueda ser sobrepasada en un sitio dado. Los osteocitos pueden transmitir mensajes mecánicos o eléctricos de áreas profundas dentro del hueso a las células superficiales de lineamiento con los que están conectadas por uniones de brecha. El transmisor de los mensajes puede ser la adenosina.

Las células de lineamiento óseo transducen los impulsos de activación al aparato de reabsorción potencial al preparar el sitio tal vez por estímulo hormonal. Las prostaglandinas E2 liberadas por las células de lineamiento pueden a su vez ayudar en el reclutamiento de los osteoclastos. Los reguladores sistémicos y locales pueden también madurar los osteoclastos; los osteoclastos son entonces reclutados y activados; los factores autocrinos y paracrinos de crecimiento originado en los osteoblastos aseguran su suministro suficiente de precursores osteoblásticos para la formación ósea (16).

La osteoporosis es una consecuencia normal del envejecimiento. La masa ósea alcanza su máximo nivel a los 30 años, empezando a declinar después de los 40 a razón de $0.5 \%$ al año. En la mujer postmenopáusica y hasta por varios años, este porcentaje puede ser de $1 \%$ o más y hasta $5 \%$ en el hueso trabecular del esqueleto axial (17).

Como recordamos, el hueso tiene una zona esponjosa o trabecular de finas espículas óseas y que se observa más en vértebras, pelvis y metáfisis de huesos largos; el hueso trabecular, muy activo metabólicamente, está cubierto por una membrana endóstica altamente celular compuesta por osteoblastos que elaboran un osteoide y la fosfatasa alcalina.La otra zona del hueso es la cortical, que se observa en la diáfisis de los huesos largos (18). La pérdida de masa ósea se hace una década más temprano en el hueso trabecular que en el cortical. Ambos tipos de huesos tienen un patrón bifásico de pérdida de masa ósea: una fase lenta que ocurre en ambos sexos y una fase acelerada transitoria que ocurre en las mujeres después de la menopausia (15).

En la osteoporosis probablemente hay tanto un aumento de la osteolisis como una disminución de la formación de hueso. Sobre estos procesos intervienen factores múltiples y heterogéneos, de origen hormonal o no. Entre los primeros están:

a) Los estrógenos, que disminuyen la respuesta osteoclástica al estímulo de la PTH.

b) Los niveles de PTH están altos en sólo una décima parte de los pacientes. La infusión del fragmento 1-34 PTH induce una respuesta insuficiente en la 1-alfa-hidroxilación renal de la vitamina $\mathrm{D}$ en los viejos osteoporóticos (19).

c) La calcitonina (CCT) tiene niveles plasmáticos menores en la mujer que en el hombre, su secreción disminuye con la edad, la respuesta del calcio a la CCT inmunorreactiva disminuye en las mujeres con la edad (20) y aunque los niveles de CCT son algo más bajos en mujeres osteoporóticas que en sanas, no hay acuerdo sobre si la osteoporosis se caracteriza por deficiencia de CCT. d) Los niveles de 1-alfa-25 (OH)2 D están disminuídos en los pacientes osteoporóticos y en los ancianos, y como vimos, hay una hiporrespuesta en la producción de calciferoles alfa hidroxilados a la infusión de PTH. Esto correlaciona con la menor absorción instestinal de calcio observada en ancianos osteoporóticos. Sin embargo, los niveles de 25-OH-D son normales (19).

Como factores no hormonales estarían la ingesta menor de calcio en el anciano, la falta de ejercicio físico de este grupo etáreo, factores raciales (la incidencia de osteoporosis es menor en los negros por ejemplo), etc.

La formación de huesos y la reabsorción ósea siguen una secuencia programada en focos discretos denominados "Unidades de Remodelación". Al principio aparecen osteoclastos en una superficie inactiva y en dos semanas construyen un túnel en el hueso cortical o una laguna en el trabecular. Los osteoclastos son remplazados por osteoblastos que llenan la cavidad reabsortiva con hueso nuevo durante tres o cuatro meses.

La mayor incidencia de fracturas en los ancianos no sólo se debe a la osteoporosis sino a una mayor propensión a las caídas por malos reflejos, mala visión, artritis, enfermedades neurológicas o ingesta de sedantes.

En el desarrollo de osteoporosis, la densidad ósea inicial juega un papel importante. Como ya habíamos mencionado, hay factores relacionados con la edad como la regulación deficiente de la actividad osteoclástica causada por las anormalidades en los factores sistémicos o locales de crecimiento, hay disminución de la absorción del calcio y otros factores hormonales relacionados con la edad y factores esporádicos como la ingesta de ciertas drogas o presencia de enfermedades que puedan inducir orteoporosis, mala o inadecuada nutrición y factores de comportamiento.

Riggs y Melton (15) han querido hablar de dos tipos de osteoporosis involutiva, la tipo I o "postmenopáusica" y la tipo II o "senil". Muchos pacientes son asintomáticos, así que es conveniente solicitar rayor $X$ laterales de columna toráxica o lumbar en mujeres de más de 40 años. El dolor lumbar es síntoma común, normalmente como resultado de fracturas en cuerpos vertebrales, fracturas que también se observan en cuello de fémur, las que son tremendamente incapacitantes. Además de las dolorosas fracturas se observará también una disminución de la estatura, citosis dorsal y pliegues cutáneos en la base del tórax.

Tabla 1

FACTORES DE RIESGO

Bien Establecidos Evidencia Moderada Evidencia no Concluyente

Obesidad (-)
Raza negra (-)
Edad (+)
Ooforectomía
Premenopáusica (+)
Corticoides (t)
Uso de estrógenos $(-)$
Inmobilidad extrema $(+)$

Alcohol (+)

Cigarrillo (+)

Ejercicio (excepto

en amenorreicas) $(-)$

Baja ingesta

de Calcio $(\rightarrow)$

Inmobilidad extrema $(+)$
Actividad moderada

Raza Asiática

Paridad

Diabetes

Tiazidas

Progestágenos

Aguas fluoradas

Cafeína
(+): Riesgo aumentado. (-): Riesgo disminuido. 
Los estudios radiológicos mostrarán desmineralización difusa, vértebras bicóncavas en "boca de bacalao" (por herniación de los núcleos pulposos), diferencias en la densidad de los cuerpos vertebrales (disminuidos) con acentuación de la cortical y de las trabéculas verticales. La altura de los cuerpos vertebrales disminuye. Se pueden observar también deformidades en cuña de los cuerpos vertebrales, aplastamientos de herniaciones localizadas en los núcleos pulposos (nódulos de Schmorl), fracturas, etc. Aunque el cráneo es a menudo respetado, se pueden ver manchas de menor densidad. La cortical se disminuye en los huesos largos. Las cifras de calcio, fósforo y fosfatasa alcalina son normales pero la calcemia puede estar aumentada (18). Los factores de riesgo que se mencionan están incluidos en la Tabla 1 (21).

La valoración de pacientes puede incluir, además de la historia y la radiología, estudios con indicadores bioquímicos (Tabla 2) y los de densitometría ósea con TAC, absorciometría de fotones de haz único o de haz doble, procedimientos no siempre disponibles y costosos. Como grupo de alto riesgo están la mujeres delgadas, de complexión ligera con un contenido mineral en el radio menor del $90 \%$ de lo esperado, con menopausia temprana, con historia familiar de osteoporosis o personal de anorexia nerviosa, alcoholismo, tabaquismo o problemas de malabsorción gastrointestinal (15).

El manejo de la osteoporosis es más que todo preventivo. Como medidas generales se sugiere el ejercicio físico y evitar las toxinas óseas (alcohol y cigarrillo).

Tabla 2

INDICADORES BIOQUIMICOS QUE SE UTILIZAN PARA IDENTIFICAR INDIVIDUOS QUE ESTAN PERDIENDO HUESO EN FORMA RAPIDA

(Modificado de Christiansen) (25)

\begin{tabular}{|ccc|}
\hline Positivo Verdadero (\%) & Variable & Falso Positivo (\%) \\
\hline 92 & * Ca/Cr Ua (mmol/mmol) & 58 \\
88 & Peso Corporal (kilo de grasa) & 54 \\
83 & $* *$ HPR-CrUA (mmol/mmol) & 39 \\
79 & Estradiol sérico (pmol/L) & 33 \\
79 & Estrona sérica (pmol/L) & 32 \\
79 & Fosfatasa alcalina sérica (U/L) & 19 \\
& & \\
\end{tabular}

* Proporción calcio/creatinina urinaria en ayunas.

** Proporción hidroxiprolina-creatinina urinaria en ayunas.

El suplemento dietético con calcio se recomienda como terapia para disminuir la pérdida ósea postmenopáusica. Los efectos colaterales posibles son mínimos, es rara la calcificación de los tejidos blandos y es remota la posibilidad de formación de cálculos (lo que puede evitarse forzando líquidos). Se pueden combinar las fuentes dietéticas (leche descremada) que contienen $1 \mathrm{mg} / \mathrm{ml}$ y los suplementos (lactato o carbonato) que suministren 1.0 a $1.5 \mathrm{~g}$ de calcio elemental por día $(18,21)$.

Los pacientes con factores de riesgo para una osteoporosis tipo I ameritarían medidas densitométricas vertebrales para iniciar tratamiento profiláctico con agentes tales como los estrógenos $(15,22,23,24)$.
El beneficio máximo derivado de esta terapia es detener o retrasar la progresión de la pérdida ósea, particularmente en la fase rápida observada en la postmenopausia inmediata (25). Un efecto lentificador de la osteolisis se ha observado con dosis bajas (19). Su uso se ha preconizado incluso en los años perimenopáusicos para tratar además los trastornos vasomotores y alivian la depresión experimentada por las mujeres estrógeno-deficientes (26). Nordin y col. (27) los usan en combinación con algún preparado de calcio y vitamina D. En los años fértiles, el uso de anovulatorios de combinación al menos por un año, tiene un efecto protector persistente (hasta 10 años después de suspendidos) contra el carcinoma de endometrio, efecto más notorio en las nulíparas, usualmente con mayor riesgo (28). En los años menopáusicos, el uso de estrógenos continuos sólo en dosis altas puede inducir una hiperplasia endometrial, la que es una lesión premaligna. Gambrell (1) mostró que al añadir un progestágeno, se reduce la incidencia de carcinoma endometrial por debajo de la vista en la población no tratada, siendo mayor este efecto protector cuando se usa por más de una semana. El mismo autor recomienda además los progestágenos, pues según sus estudios reducen el riesgo de cáncer de seno, son útiles en la prevención de la osteoporosis y promueven la formación de hueso nuevo. No hay consenso, sin embargo, en que los progestágenos añadan beneficios al efecto protector óseo de los estrógenos.

La calcitonina (CCT) sintética de salmón (que entre las de todas las especies es la CCT biológicamente más potente), ha sido usada por varios investigadores en el tratamiento de la osteoporosis. Wallach y Ziliotto por ejemplo, la han usado en forma continua hasta por dos años. Chesnut la usó también continuamente pero ha preferido asociarla con suplementos de calcio y vitamina D en dosis bajas. Según Gueguen, se requiere saber si el proceso subyacente tiene un alto nivel de remodelación ósea (osteolisis) en cuyo caso la CCT sola, serviría; pero si el proceso osteolítico (determinado por biopsia ósea, niveles de hidroxiprolina urinaria, hipocalcemia aguda inducida por CCT), es bajo, se hace necesario asociarla con otra substancia que estimule la formación ósea, tal como el fósforo (o el fluoruro). En todo caso parece más conveniente darla por períodos largos, con intervalos (tratamiento secuencial), 50 a 100 UI diariamente o tres veces a la semana; aunque hay discusión sobre la efectividad de la CCT en el tratamiento a largo plazo de la osteoporosis, muchos la usan para el tratamiento de episodios de dolor secundario al colapso vertebral en el curso de la osteoporosis generalizada, pues además de lentificar un proceso de osteolisis que se ha reactivado, la CCT tiene además un efecto analgésico (18).

Gruber y col. (33) presentaron los resultados de un estudio clínico en 45 mujeres postmenopáusicas osteoporóticas quienes fueron tratadas por dos años con carbonato de calcio $(1.200 \mathrm{mg})$ y dosis fisiológicas de vitamina D. 24 de ellas recibieron además 100 unidades de calcitonina sintética de salmón diariamente mientras las 21 restantes sirvieron de controles; las evaluaciones metabólicas practicadas a intervalos de seis a nueve meses incluyeron la determinación de calcio corporal total (por análisis de activación de neutrones), determinación de masa ósea radial, química sérica y urinaria y PTH, además de biopsias de cresta ilíaca. Sus resultados mostraron que la CCT aumentó significativa- 
mente la medida de calcio corporal total en las pacientes tratadas.

La calcitonina es costosa y requiere administración parenteral. Sin embargo, está desarrollando una preparación para usar por vía nasal.

El otro punto es el de los regímenes que estimulan la formación de huesos, ninguno de ellos aprobado por la FDA para uso en osteoporosis.

Si la enfermedad no ha avanzado mucho, existe la expectación de restaurar el hueso perdido. Con este fin, se están ensayando esquemas a base de fluoruro de sodio con calcio, el fragmento sintético 1-34 de PTH, una combinación de 1,25 (OH)2 D y fosfato (para aumentar el recambio óseo) y el estanozolol, un esteroide androgénico (18). El fluoruro sódico estimula la formación de osteoide, pero a veces la producción es de hueso pobremente mineralizado. No produce toxicidad significativa en dosis bajas, excepto dolor ocasional en tobillos y rodillas. En dosis altas, produce hiperostosis. La asociación de fluoruros de sodio con calcio, sigue siendo investigada $(34,35)$.

Los riesgos de la administración de fluoruros se limitan a intolerancia gastrointestinal, dolor osteoarticular y fracturas apendiculares. Las dosis diarias no deben sobrepasar los $50 \mathrm{mg}$, se deben preferir los preparados con cubierta entérica y usarlos más que todo en osteoporosis trabecular (36). Han sido sugeridos también los fosfatos, pues "in vitro" previenen la osteolisis osteoclástica inducida por PTH, disminuyendo además la excreción urinaria de calcio. Sin embargo, pueden conducir a un hiperparatiroidismo secundario con empeoramiento general de la enfermedad ósea.

Chesnut considera importantes los anabólicos en una terapia antiosteoporótica combinada; estas hormonas masculinas modificadas se administran es dosis de 2 a $6 \mathrm{mg}$ diarios de estanozolol o 2.5 a $5 \mathrm{mg}$ de metandrostenolona, el primero más potente y la segunda con menos efectos colaterales (efectos androgénicos, retención de líquidos, elevación de las transaminasas), que parecen estar relacionados con la dosis, son transitorios y se mejoran con la disminución de la dosis, o descontinuación de la terapia. Parece que pueden prevenir la pérdida ósea, y en cierto grado, remplazan hueso previamente perdido. El médico deberá al usarlos, poner riesgo vs. beneficio en la balanza.

Finalmente tenemos los bifosfonatos, otra clase de agentes anti-reabsortivos $(32,37)$. El etidronato disódico administrado oralmente en forma cíclica a largo plazo ha producido aumentos modestos en la densidad ósea vertebral pero más importante aún, ha llevado a una reducción significativa en la presentación de menos fracturas, cuando se le comparó con el grupo control.

\section{Aterosclerosis}

Las mujeres postmenopáusicas, al igual que las sometidas a castración (menopausia quirúrgica), tienen un aumento en el riesgo de desarrollar enfermedad coronaria (3). Durante la edad reproductiva, este riesgo es tres veces menor en las mujeres que en los hombres pero en la postmenopausia, la relación baja a 2:1, presentándose tendencia a igualar el riesgo después de los 75 años. La principal explicación que se ha dado es la del efecto de los estrógenos sobre las lipoproteínas: a) Estos aumentan las HDL, particularmente la subfracción 2 , pues disminuyen la actividad de la lipasa hepática, encargada de catalizar la hidrólisis de los fosfolípidos de las lipoproteínas, degradándolos irreversiblemente; en otras palabras, los estrógenos interfieren en la metabolización hepática de las HDL (lipoproteínas de alta densidad que transportan el colesterol hacia el hígado para excretarlo, por lo que a éste se le ha llamado el "colesterol bueno" o "protector").

b) Los estrógenos disminuyen las LDL (lipoproteínas de baja densidad ricas en colesterol aterogénico o "malo"). Las LDL interactuarán con receptores en las membranas de las células "internalizándose", para suministrar colesterol como substrato para las hormonas esteroides o para constituir las mismas membranas celulares, etc. Las LDL sobrantes son captadas por células "limpiadoras" (como los macrófagos de la íntima de la pared arterial) y de esta manera inician la placa aterosclerótica. En animales, los estrógenos ligan y aumentan la captación de partículas de LDL por el hígado.

c) Un tercer efecto de los estrógenos, el aumento de los triglicéridos (VLDL o lipoproteínas de muy baja densidad), tiene escaso o ningún efecto aterogénico porque además de haber un aumento concomitante en las HDL, las VLDL que se aumentan no son precisamente las enriquecidas con colesterol o "intermedias" (IDL) que son las aterogénicas $(38,39,40)$.

El patrón de lipoproteínas en la menopausia es entonces aterogénico (38). Este patrón se revierte en grado variable con el uso de estrógenos, dependiendo del tipo de esteroides, vía de administración, dosis, sexo, uso concomitante de progestágenos, etc. De esta manera, los estrógenos protegen contra la enfermedad coronaria, contra los accidentes cerebrovasculares y disminuyen la mortalidad por todas las causas (41-47). Los estrógenos además inhiben la formación de tromboxano (necesario para la adhesión plaquetaria), lo que interfiere con la proliferación de músculo liso arterial, que lleva a una disminución de la captación del colestero! LDL de las miofibrillas (39).

La mayoría de los estudios epidemiológicos confirman este efecto antiaterogénico de los estrógenos; los que se han usado son generalmente estrógenos equinos conjugados, los más antiguos y populares, por consiguiente los más intensamente estudiados (43); con excepción de un reporte proveniente de Framingham, que confirmó el efecto benéfico sobre las lipoproteínas pero no encontró diferencias en mortalidad cardiovascular entre usuarias y no usuarias, los demás estudios, como hemos mencionado, muestran un efecto favorable (5). El grupo de Wisconsin mostró hallazgos angiográficos en cuanto a presencia y progresión de la enfermedad que fueron significativamente mejores en usuarias que en no usuarias, dentro de un grupo de 933 mujeres menopáusicas. También se observó diferencia significativa en la concentración de HDL pero no se obtuvo información sobre si hubo o no un uso concomitante de progestágenos (42).

El punto de la mortalidadd cardiovascular en la menopausia es quizá el más importante de todos, incluso más que la prevención de la osteoporosis o de los síntomas vasomotores y genitourinarios tan molestos y la preocupación por el cáncer o por los efector colaterales. En los Estados Unidos, por ejemplo, en 1982 murieron 36.000 mujeres de cáncer 
de seno mientras que 355.000 fallecieron de enfermedad cardiovascular. En 1990 habrá más de 43 millones de ancianas mayores de 50 años. El riesgo de morir de enfermedad cardiovascular fue apenas un tercio entre las que tomaron estrógeno solo, comparadas con las que no lo tomaron, en un grupo de 2.270 mujeres. La decisión de formular los estrógenos debe sin embargo tomarse de manera individual, valorando riesgo vs. beneficio (48).

Los estrógenos intervienen en otros mecanismos que afectan la función cardiovascular; por ejemplo, corrigen la reducción en el flujo sanguíneo que está comprometida en varias etapas del desarrollo de la aterosclerosis (46). Experimentalmente, parece que los estrógenos inhiben la captación de LDL por la pared arterial, aún asociados a progestágenos (47).

Pero si los efectos benéficos cardiovasculares de los estrógenos son principalmente medidos por su acción favorable sobre las lipoproteínas, sería entonces vital que este efecto no fuese contrarrestado o parcialmente negado, como lo es, por el uso concomitante de progestágenos (45). Por ejemplo, hubo consenso reciente entre los expertos, que hay suficiente evidencia para apoyar el uso de los estrógenos solos en las postmenopáusicas histerectomizadas por un efecto cardioprotector y de que los progestágenos no tienen que usarse rutinariamente en estos casos (49). Los beneficios cardiovasculares de esta terapia única con estrógenos en pacientes sin útero sobrepasan ampliamente los riesgos. En Norteamérica un $37 \%$ de las mujeres son sometidas a histerectomía antes de los 60 años.

Los progestágenos, cuando se administran por vía oral, tienen efectos contrarios a los de los estrógenos (39). De escoger un progestágeno, el ideal desde el punto de vista de lipoproteínas es la progesterona micronizada (no disponible comercialmente); en segundo lugar estarían el acetato de medroxiprogesterona y los nuevos derivados de 19-nortestosterona, gestodene y desogestrel (45). Pero en general, el impacto tiende a ser negativo en mayor o menor grado, no sólo sobre los lípidos sino sobre el sistema prostaglandina/ tromboxano (41).

Al hacer una comparación de los cambios estimados en la mortalidad anual inducidos por la suplencia con estrógenos equinos conjugados $(0.625 \mathrm{mg}$ diarios $)$ o combinados con una dosis adecuada de progestágenos por diez días al mes, ambos tratamientos por diez años, Henderson, Ross, Lobo, Pike y Mack (50), usaron como parámetros las reducciones en fracturas de cadera, cáncer endometrial, cáncer de seno y enfermedad coronaria. La reducción neta de muertes por 100.00 pacientes fue de 230 para el grupo de estrógenos solos y de 163 para la terapia combinada en las pacientes con útero intacto; en las histerectomizadas fue de 256 para el primer grupo y de 163 para el segundo. El impacto del cambio estuvo más que todo en la reducción de la mortalidad por enfermedad coronaria, lo que los hace preferir la terapia de estrógeno solo para las histerectomizadas y el uso juicioso de la dosis menos potente de progestágeno capaz de impedir la enfermedad endometrial estrógeno-inducida.

Debe anotarse que la administración no oral de estrógenos (parche, pellets, crema) no parece tener la misma magnitud de efecto benéfico sobre los lípidos y las lipoproteínas que la oral, particularmente en el uso a corto plazo. Esto puede atribuirse a la falta de metabolismo de primer paso a niveles más constantes en contraposición a fluctuaciones diarias $(39,45)$. Las elevaciones que se observan en las HDL por rutas no orales son usualmente acompañadas de aumentos en el estradiol sérico con poco aumento en la proteína transportadora de hormonas sexuales, lo que podría hacer a estas rutas más estrogénicas para el seno que los estrógenos equinos conjugados administrados por vía oral (41). Los estrógenos en dosis altas en hombres aumentan la enfermedad coronaria (43).

\section{Estrógenoterapia: riesgos y vías de administración}

Las indicaciones y contraindicaciones de las varias alternativas de terapia estrogénica suelen ser las mismas aunque hay algunas diferencias, a veces en el plano hipotético en cuanto a su farmacocinética, indicaciones específicas y los efectos colaterales. En la primera parte de esta sección discutiremos lo relacionado con el riesgo de cáncer.

a) Cáncer Endometrial: En la década de los 70 se demostró

que la administración de estrógenos solos aumenta el riesgo de cáncer endometrial entre seis y quince veces, dependiendo tanto de la dosis como de la duración del tratamiento (51). A pesar de esta asociación, estas mujeres con cáncer endometrial inducido por estrógenos exógenos tienen sólo una pequeña reducción en su expectativa de vida comparada con las sanas, posiblemente porque tienen una mejor supervisión médica (50). La adición de un progestágeno a la terapia estrogénica reduce el riesgo de carcinoma endometrial al prevenir y/o tratar de manera efectiva la hiperplasia del endometrio. Con la asociación estro-progestágena, la incidencia de cáncer endometrial cae por debajo de la incidencia en no-usuarias de suplencia hormonal $(52,53,54$, 55). La terapia de combinación produce un patrón regular de hemorragia lo que reduce la necesidad de biopsia endometrial (55). Si se va a añadir progestágeno, las tabletas deben suministrarse durante doce a trece días para bloquear totalmente la hiperplasia endometrial. Muchos utilizan la medroxiprogesterona (53) y otros utilizan dosis bajas de $\mathrm{D} / \mathrm{L}$ norgestrel o de noretindrona (54).

b) Cáncer de Seno: No parece haber preocupación entre los

expertos por el uso a corto plazo de estrógenos y su relación con cáncer de seno mientras que a largo plazo, la evidencia no es concluyente, pues algunos estudios han reportado un aumento en la incidencia y otros no. ¿Qué es corto plazo? La mayoría diría que entre tres o cinco y diez años. Sin embargo, como un buen número de tumores malignos del seno son estrógeno-dependientes (es decir, tienen receptores estrógenicos positivos) es obvio que estas hormonas están contraindicadas en las neoplasias malignas de seno pre-existentes o sospechadas. Por otro lado, en el ciclo menstrual normal se ha visto que hay una proliferación celular máxima del epitelio mamario durante la fase luteínica (cuando hay secreción tanto de estrógenos como de progesterona) y no durante la fase folicular temprana, cuando sólo hay un aumento en los niveles de estrógenos; la fisiología puede entonces proveer una lógica al interpretar los hallazgos epidemiológicos (56). Por otro lado, los estrógenos parecen inducir tumores mamarios en animales, particularmente en cepas susceptibles de ratones (3).

La incidencia de cáncer de seno conserva su tendencia ascendente durante la postmenopausia (1). Existen también 
unos factores de riesgo: historia familiar, nuliparidad, primer embarazo tardío, historia de enfermedad benigna del seno del tipo proliferativo, menopausia tardía y menarquia precoz, entre otros, por lo que algunos autores no aconsejan el uso de estrógenos exógenos en estos casos (57); pero otros, al no observar patrones consistentes en los estudios epidemiológicos, no consideran estos factores de revelancia en las decisiones terapéuticas (56).

La mayoría de los estudios realizados para valorar el efecto cancerígeno de los estrógenos exógenos sobre el seno no han encontrado un riesgo aumentado $(58,59,60)$ y algunos han hallado una disminución en la mortalidad por cáncer de seno en las usuarias de terapia de suplencia estrogénica. Otros estudios han señalado un aumento del riesgo relativo con dosis altas por más de cinco años e historia familiar de cáncer de seno (61) o en las usuarias de un particular tipo de estrógeno (estradiol), particularmente al asociarse con progestágeno (62). Pero el análisis de los subgrupos de casos está limitado por ejemplo por el pequeño número de pacientes en estos subgrupos y se subestima el uso previo de estrógenos por un determinado número de años (63). Existe además el hecho de que si la incidencia de cáncer de seno ha aumentado en los últimos diez años en los Estados Unidos, esto se ha debido más al diagnóstico temprano por mayor tamizaje que propiamente a un aumento paralelo al uso de estrógenos, por lo que algunos consideran que el impacto que ellos tienen sobre la ocurrencia de cáncer de seno es mínimo (56). En cuanto al uso concomitante de progestágenos, las opiniones van desde una disminución de la incidencia hasta un aumento de la misma, pasando por ningún efecto protector $(1,5,49,50,56,62)$. Generalmente se estima que los riesgos de los estrógenos deben considerarse en forma global, comparándolos con los grandes beneficios con la mejoría de la calidad de vida y con la mortalidad general que estaría disminuida (50). El uso del autoexamen mensual y de la mamografía anual a partir de los 40 años redundará en el diagnóstico precoz y en mejores perspectivas pronósticas (64). Según Whitehead, hay dos escuelas: los que prefieren los estrógenos solos y los que los usan con progestágenos de manera secuencial (55). Como terapia investigativa, están además las combinaciones fijas de estroprogestágenos.

c) Otros riesgos: Sobre la base de lo que se observa con los anticonceptivos orales se ha hablado de riesgos de hipertensión, enfermedad tromboembólica y colelitiasis. Los parámetros metabólicos que pudiesen tener alguna influencia están relacionados con la vía de administración: los estrógenos orales (v. gr. los equinos conjugados) que tienen un metabolismo hepático de primer paso y los no orales (v. gr. los mismos estrógenos equinos administrados por vía vaginal o el estradiol transdérmico o "parche") que no tienen ese metabolismo hepático de primer paso (65-68). Los "parches" incrementan notablemente la concentración de estradiol sérico (41) cosa que no ocurre con los otros preparados que suministran más que todo estrona. Los estrógenos conjugados pueden incrementar la actividad de renina plasmática aunque no aumenta la concentración de la renina en sí; en general, los estrógenos pueden causar cierto grado de retención de líquidos; pero es improbable que la terapia a bajas dosis cause hipertensión significativa en la mayoría de las pacientes postmenopáusicas (3) e incluso varios estudios muestran que más bien el efecto es benéfico (1). Sin embargo, como la hipertensión es común en los adultos, puede coexistir en la postmenopausia por lo que debe ser controlada adecuadamente, pero no hay que suspender la süplencia estrogénica. En cuanto a un aumento en los fenómenos tromboembólicos, esto no se ha establecido con la estrógenoterapia oral de suplencia $(1,3)$. Tampoco hay una asociación primaria entre ésta y accidentes cerebrovasculares. Algunos cambios en los factores de coagulación se han observado con el envejecimiento y otros con la estrógenoterapia; entre estos últimos están los niveles hormonales altos de fibrinógeno, niveles normales bajos de anti-trombina III y una disminución en el factor V.Aunque un Comité Gineco-Obstétrico asesor de la FDA aconsejó un cambio en las contraindicaciones, la enfermedad tromboembólica y la tromboflebitis activa permanecen en la lista de ellos (1). No hay suficiente información en cuanto a su uso en personas con antecedentes tromboembólicos. Hammond (3) aconseja no utilizar estrógenos de ningún tipo en pacientes con factores de riesgo para tromboflebitis como trauma, cirugía, inmovilización, etc.

En cuanto a la colelitiasis, el riesgo relativo es de 2.5 en las mujeres que toman estrógenos, quizá por alteraciones en el balance lípido o en el contenido vesicular de sales biliares. Tanto los estrógenos orales como los percutáneos incluyen la colelitiasis entre sus advertencias. Son contraindicaciones relativas de los estrógenos la historia familiar de neoplasia estrógeno-dependiente, los leiomiomas uterinos, venas varicosas severas, la historia de enfermedad hepática, la diabetes mellitus, la porfiria y la hipertensión severa (3).

Los estrógenos tienen entonces varias vías de administración. La presentación inyectable no la discutiremos ahora, pues su indicación son las hemorragias uterinas disfuncionales. La vía oral está básicamente representada por los estrógenos equinos conjugados que suministran estrona y equilina o por otros preparados menos comúnmente formulados, como los diferentes compuestos a base de estradiol. La dosis usual de estrógenos equinos (naturales) conjugados es de $0.625 \mathrm{mg}$ diarios (1) con excepción de los casos de mujeres con menopausia quirúrgica o con osteoporosis establecida, donde puede necesitarse una dosis más alta, al menos en un comienzo.

La vía percutánea (o no oral) representa una alternativa e incluye la crema vaginal, la vía transdérmica (parche) y los "pellets"; esta vía omite el metabolismo de primer paso, como se dijo anteriormente. La crema (usada básicamente a corto plazo para problemas urogenitales) a niveles sistémicos, aunque con un cuarto de la dosis usual no se absorbe pero sí cambia la histología vaginal (65). El parche (69) suministra principalmente niveles de estradiol y no de estrona, se cambia dos veces por semana y puede producir irritación local. En una serie de presentaciones realizadas en un Seminario en Lake Buenavista, FL en 1984, donde se comparó básicamente el estradiol transdérmico con los estrógenos equinos conjugados (70), no se observaron diferencias en cuanto al grado de estimulación endometrial, parámetros de coagulación, cambios en las cifras tensionales, disminución de la relación calcio/creatinina, síntomas vasomotores y vaginales. Hubo diferencias en cuanto al substrato de renina, la transcortina (CBG) y la proteína transportadora de hormonas sexuales (aumentadas con la vía oral) y con el perfil lipídico ( $\sin$ cambios para el transdérmico, con 
cambios favorables para la vía oral). El metabolismo de primer paso es entonces la causa de algunos inconvenientes a veces observados con los estrógenos sintéticos de los anticonceptivos orales pero en la terapia oral de suplencia estrogénica no parecen producirse cambios de significancia clínica. Los cambios favorables en el perfil lipídico (que reducen el riesgo de enfermedad coronaria) son más que todo característicos de la vía oral, sobre todo a corto plazo y con las bajas dosis usadas con los parches, por lo que esto debe ser una consideración para tener en cuenta el escoger la vía de administración (si recordamos que la enfermedad coronaria es la principal causa de mortalidad en la postmenopausia) (68).

Resumiendo, la terapia de suplencia estrogénica tiene entonces un balance riesgo/beneficio positivo pues mejora la calidad de vida al corregir síntomas climatéricos de tipo endocrino (Tabla 3) (71). Como dicha terapia no está desprovista de riesgos, se hace necesario un seguimiento estricto de la paciente por parte del médico, con una selección previa adecuada de los casos en donde el beneficio sea tangible y no existan contraindicaciones.
Tabla 3

\section{SINTOMAS CLIMATERICOS DE NATURALEZA ENDOCRINA (71)}

\begin{tabular}{|c|c|}
\hline Organo Blanco & Posible Síntoma \\
\hline 1. Vulva, vagina & - Dispareunia. \\
\hline 2. Vejiga, uretra & $\begin{array}{l}\text {-Flujo con pintas de sangre. } \\
\text { Prurito vulvar. }\end{array}$ \\
\hline $\begin{array}{l}\text { 3. Utero, piso } \\
\text { pélvico }\end{array}$ & $\begin{array}{l}\text {-Poliaquiuria, tenesmo vesical, } \\
\text { incontinencia de estrés. } \\
\text { Prolapso útero-vaginal. }\end{array}$ \\
\hline $\begin{array}{l}\text { 4. Piel, membranas } \\
\text { mucosas }\end{array}$ & $\begin{array}{l}\text { Resequedad, prurito. Riesgo de trauma. } \\
\text { Sequedad y caída del cabello. Hirsutismo leve. } \\
\text { Boca seca. }\end{array}$ \\
\hline 5. Senos & $\begin{array}{l}\text { Tamaño reducido. Consistencia más blanda. } \\
\text { Senos caídos. }\end{array}$ \\
\hline $\begin{array}{l}\text { 6. Esqueleto } \\
\text { 7. Sistema } \\
\text { cardiovascular }\end{array}$ & $\begin{array}{l}\text { Fracturas de cadera y muñeca. Lumbalgia. } \\
\text { Angina, enfermedad coronaria. }\end{array}$ \\
\hline 8. Sistema nervioso & $\begin{array}{l}\text { Insomnio. Cambio en el temperamento. Cambios } \\
\text { en la memoria. Pérdida de la discriminación } \\
\text { de dos puntos. }\end{array}$ \\
\hline
\end{tabular}

\section{BIBLIOGRAFIA}

1. Gambrell RD. Estrogen replacement therapy, 1st. ed. Essential Med Info Systems. Armityville NY: 1987.

2. Ponte CD, Swinker ML, Madhavan S. Estrogen replacement therapy, a pilot survey of primary care physicians in West Virginia. DICP 1989; 23: 977-979.

3. Hammond CB, Maxson WS. Current status of estrogen therapy for the menopause. Fertil Steril 1982; 37: 5-25.

4. Kupperman HS. The beneficial effects of estrogen. Female Pat 1980; 5: 3-37.

5. Gambrell RD. The menopause, benefits and risks of estrogen-progestogen replacement therapy. Medical Times 1989; 117: 35-43.

6. Ginsburg J. Menopausal hot flushes. In: From menopause to osteoporosis, a challenge to modern society, MDL Pintasilgo, President of Symposium, WHO, Geneva; 1988.

7. Metzger DA, Hammond CB. Are estrogens indicated for the treatment of postmenopausal women? Drug Intell Clin Pharm 1988; 22: 493-496.

8. Figueroa-Casas PR, Schlaen I. Sequential use of conjugated estrogens and medroxiprogesterone in the climacteric syndrome, clinical and histologic findings. Maturitas 1988; 9: 309-313.

9. Ma HK. Estrogen therapy, panacea for ills of postmenopausal women. JAMA-SEA $1988 ; 4: 4-4$.

10. Tamelle R. Non-hormonal treatments of climacteric disorders. In: From menopause to osteoporosis, a challenge to modern society. MDL Pintasilgo, President of Symposium, WHO, Geneva; 1988.

11. Council on scientific affairs. Estrogen replacement in the menopause. JAMA 1983; 249: 359-361

12. Hammond CB, Quigley MM. Use of sex steroids in clinical management of the menopause. In: Current Problems in Obstetrics and Gynecology (JM Leventhal, ed.), Yearbook Med Publ, Chicago 1981; 4: 17-37.

13. Comité Editorial. Estrógenos, menopausia y sexualidad. Iladiba, junio $1990 ; 59-60$

14. Symposium review. From menopause to osteoporosis, a challenge to modern society (ML Pintasilgo, President), WHO, Geneva, May 1988; $1-24$.

15. Riggs BL, Melton LJ. Involutional osteoporosis. N Eng J Med 1986; 314: 1676-1686.

16. Peck WA, Woods WL. The cells of bone. In: Osteoporosis, etiology, diagnosis and management (BL Riggs, LJ Melton, eds.), Raven Press, NY 1988; 1-44.
17. Whedon GD. Osteoporosis (editorial). N Eng J Med 1981; 305: 397-399.

18. Jácome-Roca. Fisiopatología paratiroidea, calcitonina, calciferoles y metabolismo óseo. Gráficas Esfera, Bogotá 1985; 57-61.

19. Slovick DM, Adams JS, Neer RM, Holick MF, Potts JJ. Deficient production of 1-alfa-25, dihydroxivitamin D in elderly osteoporotic patients, N Eng J Med 1981; 305: 372-374.

20. Austin LA, Heath H. Calcitonin physiology and pathophysiology. N Eng J Med 1981; 304: 269-277.

21. Peck WA, Riggs BL, Bell NH, Wallace RB, Johnston CC, Gordon SL, Shulman LE. Research directions in osteoporosis. Amer J Med 1988; 84: 275-282.

22. Lindsay R. Prevention and treatment of osteoporosis with ovarian hormones. Ann Chir Gynaecol 1988; 77: 219-223.

23. Nordin BEC. Treatment of postmenopausal osteoporosis. Drugs 1979; 18: 484-492.

24. Weiss NS, Ure CL, Ballard JH, Williams AR, Daling JR. Decreased risk of fractures of the hip and lower forearm with postmenopausal use of estrogen. N Eng J Med 1980; 303: 1195- 1198.

25. Christiansen C, Christiansen MS, Mc Nain P, Hager C, Stockhanc $\mathrm{K}$, Transbol IB. Prevention of early postmenopausal bone loss, controlled two-year study in 315 normal females. Europ $\mathbf{J}$ Clin Invest 1980; 10: 273-279.

26. Wells RG. Suplencia hormonal antes de la menopausia. Trib Med Col 1990; 82: 56-61.

27. Nordin BEC, Horsman A, Crilly RG, Marshall DH, Simpson M. Treatment of spinal osteoporosis in postmenopausal women. Brit Med J 1980; 1: 451-454.

28. Ory HW (CDC Cancer and steroid hormone study). Oral contraceptive use and the risk of endometrial cancer. JAMA 1983; 249: 1600-1604.

29. Mc Donall PC. Estrogen plus progestin in postmenopausal women (editorial). N Eng J Med 1981; 305: 1649-1645.

30. Weinstein MC. Estrogen use in postmenopausal women, costs, risks and benefits. N Eng J Med 1980; 303: 315.

31. Lindsay R. Estrogen treatment of patients with established postmenopausal osteoporosis. Obst \& Gynecol 1990; 76: 290-295.

32. Riggs BL. A new option for treating osteoporosis (editorial). N Eng J Med 1990; 323: 124-125.

33. Gruber HE, Irey JL, Bavlink DJ, Mattews M, Nelp WB, Sisom K, 
'Chesnut CH. Long-term calcitonin therapy in postmenopausal osteoporosis. Metabolism 1984; 32: 295-303.

34. Kleerekoper M. Effect of fluoride on osteoporosis. Consultant 1990; 30 (suppl.): 50-54.

35. Riggs BL, Hodgson SF, O'Fallon WM, et al. Effect of fluoride treatment on the fracture rate in postmenopausal women with osteoporosis. N Eng J Med 1990; 322: 802-809.

36. Kannis JA. Effect of fluoride on postmenopausal osteoporosis (letter). N Eng J Med 1990; 323: 416.

37. Editorial. New treatments for osteoporosis. Lancet 1990; 335 1065-1066.

38. Matthews KA, Meilatin E, Kuller LH, Kelsey SF, Cagginla AW, Wing RR. Menopause and risk factors for coronary heart disease. N Eng J Med 1989; 321: 641-646.

39. Burkman RT. Lipid and lipoprotein changes in relation to oral contraception and hormonal replacement therapy. Fertil Steril 1988; 49: 395-505.

40. Samsiae G. Effects of estrogen on various lipid constituents. Consultant 1990; 30: 58-62.

41. Pike MC, Henderson BE, Mack TM, Lobo RA, Ross RK. Stroke prevention and oestrogen replacement (letter). Lancet II 1989; 1034-1035.

42. Gruchow HW, Anderson AJ, Barborick JJ, Sobocinski KA. Postmenopausal use of estrogen and occlusion of coronary arteries. Amer Heart J 1988; 115: 954-963.

43. Ross RK, Paganini-Hill A, Mack TM, Arthen M, Henderson BE. Menopausal oestrogen therapy and protection from death from ischaemic heart disease. Lancet I 1981; 858-860.

44. Bush TL, Cowan LD, Barrett-Connor E, Criqui MH, Karon JM, Wallace RB, Tyroler HA, Rifkind BM. Estrogen use and all-cause mortality, preliminary results from the lipid research clinics program follow-up study. JAMA 1983; 249: 903-906.

45. Crona NM. Effects of progestins on lipids. Consultant 1990; 30 72-74.

46. Sarrel PM. Other mechanisms affecting cardiovascular function. Consultant 1990; 30: 63-66.

47. Adams MR, Wagner JD, Clarkson TB. Arterial effects of estrogen and progestin. Consultant 1990; 30: 75-77.

48. Medical News. Hormone replacement, gynecologists consider the heart of the matter. JAMA-SEA 1988; $4: 7$.

49. Consensus conference. Progestagen use in postmenopausal women. Lancet II 1988; 1243-1244.

50. Henderson BE, Pike MC, Ross RK, Mack TM, Lobo RA. Re-evaluating the role of progestogen therapy after the menopause. Fertil Steril 1988; 49 (suppl.): 95-145.

51. Antunes CMF, Stolley PD, Rosenshein NB, et al. Endometrial cancer and estrogen use, report of a large case-control study. N Eng J Med 1979; 300: 9-13

52. Gambrell RD. Prevention of endometrial cancer with progestogens. Maturitas 1986; 8: 159-168.
53. Figueroa-Casas, Schlaen I. Sequential use of conjugated estrogens and medroxiprogesterone in the climacteric syndrome, clinical and histologic changes. Maturitas 1988; 9: 309-313.

54. Whitehead MI, Townsend PT, Pryse-Davies J, Ryder TA, King RJB Effects of estrogens and progestins on the biochemistry and morphology of the postmenopausal endometrium. N Eng J Med 1981; 305: 1599-1605.

55. Whitehead MI. Role of progestins in estrogen replacement therapy, overview. Consultant 1990; 30 (suppl.): 67-68.

56. Thomas DB. The impact of estrogen on the breast. Consultant 1990; 30 (suppl.): 19-23.

57. Abaunza H. Etiología. En: "Cáncer Mamario" (H. Abaunza, ed.). Fund. Ofa, Bogotá 1987; 19-34.

58. Kanfman DW, Miller DR, Rosemberg L, Helmirich SO, Stolley P, Schfsenfeld D, Shapiro S. Noncontraceptive estrogen use and the risk of breast cancer. JAMA 1984; 252: 63-67.

59. Editorial. Hormone replacement therapy and cancer, is there cause for concern? Lancet II 1989; 368-369.

60. Young RL, Goldzieher JW. Current status os postmenopausal estrogen therapy. J Paed Obst Gynecol 1988; March/April: 4-15.

61. Hoover R, Glass A, Finkle WD, Azevedo D, Milne K. Conjugated estrogen and breast cancer risk in women. JNCI 1981; 67: 815-820.

62. Bergkvist L, Adami HO, Persson I, Hoover R, Shairer C. The risk of breast cancer after estrogen and estrogen-progestin replacement N Eng J Med 1989; 321: 293-297.

63. Barrett-Connor E. Postmenopausal estrogen replacement and breast cancer (editorial). N Eng J Med 1989; 321: 319-320.

64. Sánchez H. Mamografía. En: "Cáncer Mamario" (H. Abaunza. ed.). Fund. Ofa, Bogotá 1987; 91-138.

65. Furuhjelm M, Karlgren E, Carlstrom K. Intravaginal administration of conjugated estrogens in premenopausal and postmenopausal women. Int J Gynecol Obstet 1980; 17: 335-339.

66. Laufer LR, De Fario JL, Lu JKH, et al. Estrogen replacement therapy by transdermal estradiol administration. Am J Obstet Gynecol 1983 146: 533-541.

67. Editorial. Patch up the menopause. Lancet I 1988; 861-862

68. Stampfer MJ, Sacks FM. Patch up the menopause (letter). Lancet I 1988.

69. Stevenson JC, Cust MP, Gangar KF, Hillard TC, Lees B, Whitehead MI. Effects of transdermal versus oral hormone replacement therapy on bone density in spine and proximal femur in postmenopausal women. Lancet 1990; 336: 265-268.

70. Notelovitz M. Treatment of postmenopausal women with transdermal delivery of estradiol (Luncheon Seminar, Lake Buenavista, FL) November 1984 .

71. Utian WH. Risk/benefit analysis, quality of life enhancement. Consultant 1990; 30 (suppl.): 10-13. 